\title{
Cardiac Doppler Flow Velocities in Fetuses of Well- Controlled Insulin-Dependent Diabetic Mothers
}

\author{
Gilles Plourde*, M.D., Ph.D.
}

* To whom correspondence should be addressed: Department of Diagnostic Radiology, Montreal General Hospital, McGill University, 1650 Cedar Ave., Montreal, QC, Canada H3G 1A4

\section{ABSTRACT}

The advent of Doppler echocardiography has permitted noninvasive examination of the fetal heart. In this study, computer-assisted cardiac Doppler flow velocity waveform (DFVW) analysis was used to determine whether diastolic dysfunction is manifest in the fetuses of diabetic mothers. Diastolic atrioventricular (AV) valve flow was measured in the fetuses of 40 women with well-controlled insulindependent diabetes mellitus (IDDM) at 18 to 22 weeks (timepoint A) and 30 to 35 weeks (timepoint B) gestation. These subjects were compared to 40 age-matched pregnant non-diabetic controls, and their maternal glycosylated hemoglobin HbA1c fractions (\%) were measured to gauge the adequacy of glycemic control. HbA1c fractions showed no statistically significant difference between timepoints A and $B(4.85 \pm 0.2 \%$ and $5.16 \pm 0.3 \%$, respectively), and demonstrated a well-controlled diabetic state. At timepoint $A$, the peak velocity of rapid ventricular filling $[\mathrm{E}(\mathrm{cm} / \mathrm{s})]$ through both $\mathrm{AV}$ valves, the peak velocity of atrial contraction $[\mathrm{A}(\mathrm{cm} / \mathrm{s})]$ through the tricuspid valve, and the flow integral measurement for atrial contraction $[\mathrm{AI}(\mathrm{cm})]$ through the tricuspid valve were significantly $(p<0.05)$ higher than those measured in the controls. Peak velocities of atrial contraction and rapid ventricular filling (A and E, respectively) for both valves, as well as the flow integral of rapid ventricular filling (EI) for the tricuspid valve, were significantly $(p<0.05)$ higher in fetuses of diabetic mothers at timepoint B than in controls. Overall, despite the fact that both IDDM and non-diabetic mothers showed significant $(p<0.05)$ fetal increases in rapid ventricular filling $(E)$ between the two timepoints, the ratios $E / A$ and EI/AI for both valves were not modified in the two study groups at either gestational age. These results therefore substantiate rare previous findings that only peak velocity of rapid ventricular filling $(E)$ is increased, despite expected changes in E/A and EI/AI ratios, with gestational age. Furthermore, the results of the present study suggest that fetal diastolic function is not adversely affected in wellcontrolled maternal IDDM.

\section{INTRODUCTION}

Until recently, the diagnosis of heart defects in critically-ill neonates could only be made through invasive techniques such as cardiac catheterization and angiography. The advent of Doppler ultrasonography has had a major impact on pediatric cardiology by making non-invasive examination of the fetal heart and circulation 
possible. Simple echocardiography provides two-dimensional images of cardiac anatomy in the fetus, permitting identification of abnormalities in ventricular size, in wall thickness, and in atrioventricular (AV) or semilunar valve excursions. The addition of Doppler analysis to ultrasonography allows quantitative analysis of both velocity and direction of blood flow in the heart, and this serves as a valuable tool in the diagnosis of more subtle abnormalities in fetal cardiac circulation.

Infants of diabetic mothers represent one subgroup which unfortunately experiences an elevated risk of congenital heart anomalies. Studies of the newborns of diabetic mothers have demonstrated a five-fold increase in the $0.8 \%$ incidence of congenital cardiac defects seen in infants of non-diabetic women (1). Transposition of the great arteries, coarctation of the aorta, and ventricular septal defects (VSD) comprise the majority of the abnormalities in these neonates $(1,2)$. It is now possible to detect these anomalies at 20 weeks gestation using fetal echocardiography (2).

Previous research has shown that poorly-controlled maternal diabetes mellitus can result in fetal hyperinsulinemia with subsequent macrosomia, hypoglycemia, and ultimately, asymmetric septal hypertrophy (3). However, the risk in well-controlled diabetic mothers is less clear. Insulin normally stimulates fetal growth in the third trimester through its regulation of glycogen, fat, and protein synthesis (4); thus, hyperinsulinemic fetuses often experience exaggerated weight gain. Similarly, the fetal heart, rich in insulin receptors (5), may undergo increased ventricular septal thickening in the presence of high insulin levels in the third trimester, culminating in asymmetric septal hypertrophy (6-8). Asymmetric septal hypertrophy, found at increased incidence in infants of insulin-dependent diabetic mothers, often hinders diastolic function, as evidenced by decreased cardiac output and increased morbidity, including persistent pulmonary hypertension of the newborn, and perhaps idiopathic respiratory distress syndrome $(1,2,8)$. Simple echocardiography can detect this septal hypertrophy only at 31-34 weeks of gestation (3); however, pathological changes in cardiac circulation may occur earlier. To detect these changes, blood flow velocities through fetal valves can be measured using the addition of Doppler analysis.

Prior studies using Doppler echocardiography to examine normal fetal development have shown that early in gestation, the flow velocity profile through the AV valves is characterized by a lower early peak (E) than late peak (A) velocity wave form, the former representing ventricular muscle relaxation (i.e., filling) and the latter, atrial contraction. This decreased ratio of E/A observed early in gestation appears to signify decreased compliance of the fetal myocardium (9). Interestingly, with advancing gestational age, this ratio normally undergoes a progressive increase, representing improved myocardial compliance with maturation (10), and thus enhanced diastolic function.

This study is the first to use computer-assisted Doppler ultrasonography to determine the aforementioned parameters of cardiac function in the fetuses of mothers with well-controlled insulin- dependent mellitus (IDDM). By obtaining measurements of blood flow velocity through the fetal mitral and tricuspid valves at both 18-22 weeks and 30-35 weeks of gestation, the normal physiologic modifications in fetal diastolic function observed during maturation are investigated, as well as the impact of well-controlled IDDM on fetal diastolic function.

\section{MATERIALS AND METHODS}

\section{Subjects}

The study group consisted of 40 pregnant insulin-dependent diabetic women, mean age $28.2 \pm 5.4 \mathrm{yr}$, referred for consultation to the Pediatric Cardiology Service of Sainte-Justine Hospital Center, Montreal, Quebec. Forty age-matched pregnant non-diabetic women were randomly selected from a clinic patient population to serve as the control group. Both groups gave their written consent to participate in the study, whose protocol was approved by the medical ethics committee of the Sainte-Justine Hospital. Initial referral occurred at 18 to 
22 weeks of gestation. All the women selected underwent complete follow-up examinations until delivery.

\section{Maternal Glycosylated Hemoglobin (HbA1c) Fraction Levels}

Maternal glycosylated hemoglobin (HbA1c) fraction levels, obtained routinely during the gestation period, were used as an index of the quality of maternal diabetic control. The HbA1c level was determined using high-performance liquid chromatography as described by Nathan et al (11). An HbA1c of 5.5\% or greater reflected inadequate control of glucose levels.

\section{Doppler Flow Velocity Waveform (DFVW) Measurements}

Doppler echocardiographic studies were performed either with an HP Sonos 500 model 77020 AC or Sonos 100 model 77570 B equipped with a 3.5 or $5 \mathrm{MHz}$ transducer, as described previously (12). Briefly, DFVW through the atrioventricular valves were recorded in a four-chamber view. To obtain accurate velocities through the valves, data were only recorded from patients in whom it was possible to position the ultrasound beam parallel to or at an angle of $<30^{\circ}$ from the presumed orientation of blood flow. The sample volume was placed on or a few millimeters beyond the valve annulus. Three maximum diameter measurements of each valve were obtained by the same examiner and then averaged. The cross-sectional area of each valve was then calculated using the formula: area $=1 / 4 \mathrm{r}^{2}$. Three to five consecutive DFVW complexes at a speed of 100 $\mathrm{mm} / \mathrm{s}$ were selected and averaged for each of the two valves, and then analyzed using software previously validated in our laboratory (13). On each complex, the following variables were measured or calculated: early peak velocity $[\mathrm{E}(\mathrm{cm} / \mathrm{s})]$, late peak velocity $[\mathrm{A}(\mathrm{cm} / \mathrm{s})], \mathrm{E} / \mathrm{A}$ ratio, early flow velocity integral $[\mathrm{EI}(\mathrm{cm})]$, late flow velocity integral $[\mathrm{AI}(\mathrm{cm})]$, and EI/AI ratio.

\section{Statistical Analysis}

Results are presented as mean values \pm SD. For both gestational ages, the mean values of the aforementioned Doppler variables obtained in the diabetic women were compared to controls using an unpaired Student's $t$ test. A one-way ANOVA for repeated measures and an a posteriori Duncan multiple range test were used to determine the effect of the length of gestation on maternal HbA1c fraction (in diabetic women) and on specific Doppler variables (in both diabetic and non-diabetic women). A value of $p<0.05$ was considered statistically significant.

\section{RESULTS}

Levels of the $\mathrm{HbA} 1 \mathrm{c}$ fraction measured in diabetic mothers did not statistically differ between the two gestational ages, with values of $4.85 \pm 0.2 \%$ and $5.16 \pm 0.3 \%$ for timepoints $\mathrm{A}$ and $\mathrm{B}$, respectively. Both values indicated well-controlled glycemia.

Representative echocardiograms of DFVW analysis of fetal AV valves are shown in Figures 1 and 2. The results of the DFVW analysis of fetal valves in both IDDM and non-diabetic mothers of 18-22 weeks gestational age are presented in Table 1. The data show that ventricular filling (E) values for both the mitral and tricuspid valves are significantly raised in fetuses of diabetic women compared to those of non-diabetic women. However, the atrial contraction (A) values are significantly higher only for the tricuspid valve of the fetuses of diabetic women. Similarly, the late flow velocity integral (AI) through the tricuspid valve, but not the mitral, is significantly increased in fetuses of diabetic women. At either gestational age, the values for the early flow velocity integral (EI), the ratio of ventricular filling over atrial contraction (E/A), and the ratio of the early over late flow velocity integrals (EI/AI) for either valve did not differ significantly between the fetuses of IDDM and non-diabetic women.

The results of the DFVW study of fetal mitral and tricuspid valves at 30-35 weeks gestational age in both 
diabetic and non-diabetic (control) mothers are presented in Table 2. The data show that ventricular filling (E) and atrial contraction (A) for both AV valves are significantly higher in fetuses of diabetic women. The late flow velocity integral (AI) for the mitral valve and the early flow velocity integral (EI) for the tricuspid valve are also significantly increased in fetuses of diabetic women. Yet, when expressed as the ratio of ventricular filling over atrial contraction (E/A) or the ratio of the early over late flow velocity integrals (EI/AI), there was no significant difference between diabetic and control groups.

A one-way ANOVA for repeated measures and an a posteriori Duncan multiple range test were performed on the following variables: E, A, E/A and EI/AI. In accordance with previous work $(9,12)$, results of this study demonstrate an increase in ventricular filling velocity waveform (E) with advancing gestational age for fetuses of both IDDM and non-diabetic mothers.

\section{DISCUSSION}

This study is the first to report on the Doppler flow velocity waveforms through the AV valves of fetuses of IDDM mothers, and on the physiologic modifications in fetal diastolic cardiovascular function during the progression of gestation in diabetic women. From a theoretical point of view, human fetal echo-cardiographic Doppler studies differ from those performed on postnatal subjects in two principal respects. First, the fetal heart's right and left ventricles both eject into the systemic circulation in parallel. The output of the left ventricle is directed through the ascending aorta to the upper body organs, including the brain and heart, whereas the output of the right ventricle is directed to the pulmonary circulation and through the patent ductus arteriosus to the lower body and placenta (14). In the human fetus, right ventricular output typically exceeds left ventricular output, resulting in a right-to-left ratio of ventricular output greater than one $(15,16)$. Secondly, fetal cardiac inflow also differs from that observed in postnatal life. Blood from the fetal vena cava is directed to the left atrium, through the patent foramen ovale, and into the ascending aorta. Another stream of blood is directed through the tricuspid valve into the right ventricle, and into the pulmonary artery, with most of the flow continuing across the ductus arteriosus into the ascending aorta (17).

In the present study, the peak filling velocities across the tricuspid valve are higher than those across the mitral valve. This is consistent with the right heart dominance previously described in normal fetal animals and humans $(15,18)$. The results also demonstrate, in both IDDM and non-diabetic mothers, an increase in all measured Doppler values between the two fetal gestational ages. These findings are in accordance with those of previous investigations reporting a linear increase in these measurements with advancing gestational age $(12,19)$ and weight $(19)$.

Based on this information, a model for the changes in heart function during the progression of gestation can be hypothesized (12). During early gestation, poor ventricular relaxation results in a smaller atrioventricular gradient and, consequently, less blood enters the ventricle in early diastole. Therefore, a greater volume of blood remains in the atrium at the time of atrial contraction, creating an elevated AV gradient, and a higher atrial contraction (A) peak velocity. Improvement in ventricular relaxation, resulting in a progressively increased active ventricular muscle relaxation (E) peak without corresponding changes in atrial contraction (A), accompanies fetal maturation and extends into the first weeks after birth. With a greater volume of blood entering the ventricle during early diastole, there will be a progressive increase in both the early flow velocity integral (EI) and the early-to-late flow velocity integral (EI/AI) ratio, approaching the pattern seen at birth (20).

In accordance with previous findings (12), the results of the present study show that fetal ventricular filling (E) is significantly higher in both groups of women as gestation proceeds; however, there are no significant changes in the ratios of ventricular filling to atrial contraction (E/A) and early-to-late flow velocity integral $(\mathrm{EI} / \mathrm{AI})$ ratios during the progression of gestation in either group of mothers. 
At 18-22 weeks of gestation, ventricular filling (E) for both AV valves is significantly higher in fetuses of diabetic women than non-diabetic women. Yet, both atrial contraction (A) and the late flow velocity integral (AI) are only significantly increased for the tricuspid valve of fetuses of diabetic women. The dominance of the late diastolic component of the AV valve inflow in the fetuses of diabetic women, primarily in the right side of the heart, may suggest a decreased diastolic compliance of the right ventricle in early gestation. However, the physiological significance of this observation cannot be assessed on the basis of the present data. It should be noted that there is a high incidence of VSD in fetuses, which could conceivably result in filling defects in both sides of the heart. Since the right heart is normally dominant in fetuses, there may be an increased incidence of blood inflow anomalies in this side of the heart. Clearly, further studies are indicated in order to better understand the significance of this observed increased late diastolic waveform (A) and to explain its presence primarily on the right side.

The DFVW results at 30-35 weeks gestation show that $\mathrm{E}$ and $\mathrm{A}$ for both the mitral and tricuspid valves are significantly higher in fetuses of diabetic women than non-diabetic women. Furthermore, AI for the mitral valve and EI for the tricuspid valve are significantly elevated in fetuses of diabetic women. This may represent an improvement of right ventricular compliance with advancing gestational age, and is likely related to enhanced diastolic function occurring as part of the normal fetal maturation process.

These data support the aforementioned concept that the predominant peak velocity of atrial contraction (A) found during fetal life is essentially related to a lack of compliance in the ventricle, and may explain the importance of atrial systole in maintaining normal cardiac function in the fetus (21-23). The peak velocity of rapid ventricular filling (E) represents active ventricular muscle relaxation, but the peak velocity of atrial contraction (A) is also influenced by ventricular compliance. Thus, the respective velocity profiles of $\mathrm{E}$ and $\mathrm{A}$ are interrelated.

Conversely, aspects of this investigation do not agree with the currently-accepted notion that changes in fetal $\mathrm{E} / \mathrm{A}$ or EI/AI ratios with fetal maturation are specifically related to an improvement in ventricular compliance. In young fetuses of diabetic women, the late diastolic component of the atrioventricular valve inflows is prominent, mainly on the right side of the heart, suggesting decreased diastolic compliance of the right ventricle in early gestation. As with control fetuses, this compliance improves with fetal maturation. Therefore, the results of the present study suggest that fetal diastolic function is not adversely affected in well-controlled maternal diabetes mellitus. However, because DFVW was only examined through the AV valves, future studies measuring these parameters through the semilunar valves are indicated. In addition, further work investigating systolic function is necessary in order to better understand the potential effect of well-controlled diabetes mellitus on cardiac morbidity in newborns of diabetic mothers.

\section{ACKNOWLEDGMENTS}

The author would like to thank Dr. Jean-Claude Fouron and Dr. Joaquim Miro for their supervision, and Manon Lessard, Francine Proulx, and Alain Nadeau for technical assistance. This study was supported by the Medical Research Council of Canada and a grant from Le Fonds de la Recherche en Santé du Québec.

\section{REFERENCES}

1. Hirschfeld SS, Fanaroff AA, Merkatz IR. Cardiovascular abnormalities in infants of diabetic mothers. In: Merkatz IR, Adam PAJ, eds. The diabetic pregnancy: a perinatal perspective. New York: Grune and Stratton; 1979: 249-260.

2. La Marca E, De Geeter B. Coeur foetal et diabète maternel. In: Cardiologie pédiatrique et Foetal. Strasbourg, France; 1988: 102-108. 
3. Cooper MJ, Enderlein MA, Tarnoff H, et al. Asymmetric septal hypertrophy in infants of diabetic mothers. Fetal echocardiography and the impact of maternal diabetic control. American Journal of Diseases of Children 146(2): 226-229; 1992.

4. Joassin G, Parker ML, Pildes RS, Cornblath M. Infants of diabetic mothers. Diabetes 16(5): 306-311; 1967.

5. Devaskar SU, Szewczyk K, Devaskar UP. The fetal heart insulin receptor responds differently to varying plasma insulin concentrations. Developmental Pharmacology \& Therapeutics 10(3): 153-162; 1987.

6. Maron BJ, Verter J. Disproportionate ventricular septal thickening in the developing normal human heart. Circulation 57(3): 520-526; 1978.

7. Allan LD, Joseph MC, Boyd EG, et al. M-mode echocardiography in the developing human fetus. British Heart Journal 47(6): 573-583; 1982.

8. Veille JC, Sivakoff M, Hanson R, et al. Interventricular septal thickness in fetuses of diabetic mothers. Obstetrics and Gynecology 79(1): 51-54; 1992.

9. Reed KL, Sahn DJ, Scagnelli S, et al. Doppler echocardiographic studies of diastolic function in the human fetal heart: changes during gestation. Journal of the American College of Cardiology 8(2): 391-395; 1986.

10. Hata T, Hata K, Ryokoh K, et al. Fetal cardiac hemodynamics assessed by two-dimensional Doppler echocardiography. Asia-Oceana Journal of Obstetrics \& Gynaecology 14(1): 111-115; 1988.

11. Nathan DM, Singer DE, Hurxthal K, et al. The clinical information value of the glycosylated hemoglobin assay. New England Journal of Medicine 310(6): 341-346; 1984.

12. Carceller-Blanchard AM, Fouron JC. Determinants of the Doppler flow velocity profile through the mitral valve of the human fetus. British Heart Journal 70(5): 457-460; 1993.

13. Miro J, Rivard F, Cartwright D, et al. Computerized measurement of Doppler flow velocity waveforms: validation of a new system. Journal of the American Society of Echocardiography 5(5): 489-496; 1992.

14. Hornberger LK, Weintraub RG, Pesonen E, et al. Echocardiographic study of the morphology and growth of the aortic arch in the human fetus. Observations related to the prenatal diagnosis of coarctation. Circulation 86(3): 741-747; 1992 .

15. Reed KL, Meijboom EJ, Sahn DJ, et al. Cardiac Doppler flow velocities in human fetuses. Circulation 73(1): 41-46; 1986.

16. Allan LD, Chita SK, Al-Ghazali W, et al. Doppler echocardiographic evaluation of the normal human fetal heart. British Heart Journal 57(6): 528-533; 1987.

17. Hatle L, Angelsen B, eds. Doppler ultrasound in cardiology--physical principles and clinical applications. 2nd ed. Philadelphia, PA: Lea and Febiger; 1985.

18. Rudolph AM. Fetal and neonatal pulmonary circulation. Annual Review of Physiology 41: 383-395; 1979.

19. Saxena KK, Jose JV, Chacko J, et al. Intracardiac blood flow velocities and cardiac output in normal fetuses: a prospective pulsed Doppler echocardiographic study. Indian Heart Journal 44(6): 399-402; 1992. 
20. Areias JC, Meyer R, Scott WA, et al. Serial echocardiographic and Doppler evaluation of left ventricular diastolic filling in full-term neonates. American Journal of Cardiology 66(1): 108-111; 1990.

21. Devereux RB. Left ventricular diastolic dysfunction: early diastolic relaxation and late diastolic compliance. Journal of the American College of Cardiology 13(2): 337-339; 1989.

22. Thomas JD, Weyman AE. Echocardiographic Doppler evaluation of left ventricular diastolic function. Physics and physiology. Circulation 84(3): 977-990; 1991.

23. Zoghbi WA, Bolli R. The increasing complexity of assessing diastolic function from ventricular filling dynamics. Journal of the American College of Cardiology 17(1): 237-238; 1991.

\section{BIOGRAPHY}

Gilles Plourde, M.D., Ph.D., received his B.Sc. in Physical Activity Sciences and his M.Sc. in Nutrition from Laval University (Quebec, Quebec, Canada) in 1986 and 1989, respectively. He received his Ph.D. degree in Experimental Medicine from Laval University in 1993, and his M.D. degree from the University of Montreal (Montreal, Quebec, Canada) in 1995. He is presently a first-year diagnostic radiology resident at McGill University (Montreal, Quebec, Canada). His research on fetal cardiology was conducted during his third year of medical school as part of his summer work at the Fetal Cardiology Unit, Department of Pediatrics, Sainte-Justine Hospital (Montreal, Quebec, Canada).

Copyright (C) 1996 by MJM 